\title{
Stefan Braum
}

\section{Das Graffiti-Bekämpfungsgesetz und der Schutz des Eigentums}

Strafbar als Sachbescbädigung sind nach einer sich erneut festigenden $O L G$ Rechesprechung nur solche Graffitis, die sich niche rückstandlos entfernen lassen (Substanzverletzungstheorie). Ist dies der Fall, bleibt nuy der aufwendige Zivilrechtsweg. Es liegt daber auf der Hand, eine neue Form weichen Strafund Strafverfabrensrechts zu ersinnen, um die meist jungen Tater anzuceigen, zugleich aber über einen Täzer-Opfer-Ausgleich oder eine Wiedergutmachungsleistung lediglich das zu erzwingen, was zivilrechtlich so schwer $z$ w erreichen ist. Der Verfasser zeigt historisch die Kontinuitat eines strafrechtlichen Denkens auf, das schon immer nicht viel von klaren Grenzen der Strafbarkeit gehalten hat, das aber unter heutigen Verbältnissen zu einer neuen Qualität eon strafbewebrem Zivilvecht fubrt. Strafrechtliche Diversionsstrategien ersetzen Vollzingsdefizite des Zivilrechts.

Die Red.

\section{A. Einfübrung - Standards der Strafgesetzgebung}

*Verbrechen ist jede Handlung, weiche nach den jeweiligen Interessen des Staates unter Strafe gestellt ist. Sie braucht durchaus nicht gegen Menschheitspflichten zu verstoßen. Niche nur für die Menschbeit bedeutsame, sondern auch ganz unbedcutsame Handlungen können Delikte werden. Es steht nichts im Wege, das Rauchen auf der Straße oder das Spazierengehen in Trupps mic Strafe zu bedrohen (...)...' In dicsem Satz Wachenfelds zu Beginn des 20. Jahrhunderts scheint das aktuelle Programm der Stralgesetzgebung bereits vorgezeichnet. Seine konformistische Botschaft ist radikaler als manche Kricik, die am Strafrechr geäußerc wird. Kriminalisierung har keine Grenzen, Gesctz ist unmittelbares Resultat staatlicher Machtpolitik, kriminalpolicische Ziele sind beliebig wählbar. Noch isr die auch aus den Reihen der Strafrechtswissenschaft vorgetragene Kritik an dem 6 . Strafrechtsreformgesetz nicht völlig verhall, schon scheint sich der Gesetzgeber zu neuen kriminalpolitisclien Feldzügen anzuschicken. Graffiti und der Tatbestand der Sachbeschädigung $(\$ 30\} \mathrm{StGB})$ stehen nun im Fokus gesetzgeberischer Bemühungen.

Im Gesetzgebungsverfahren befindet sich ein Gesetzesentwurf des Bundesrates für ein "Graffiti-Bekämpfungsgesetz*. ${ }^{2}$ Der Bundesratsentwurf ist mit einem Gesetzesvorschlag der CDU/CSU-Bundescagsfraktion' weitgehend deckungsgleich. Auch dic F.D.P. unterbreiter in einem "Entwurf eines Geseizes zum verbesserren Schurz des Eigentums " einen Vorschlag zur Neufassung des $\S_{30} \mathrm{~S}_{1} \mathrm{~GB} .{ }^{4} \mathrm{Im}$ Bundesratsentwurf werden die Tathandlungen "beschädigen oder zerstören« um die Tachandlung "verunstalten $\approx$ ergänzt. Der F.D.P.-Entwurf kriminalisiert gleichfalls die Verunstalrung

I Siche F. Wachenfeld, Lehrbuch des Deuschen Seralecehes, 1914, S.69.

2 BT-Drucks. 1 / $/ 873$; kritische Auscinanderset7ung mir den Enrwivicn bei U. Bchm, SiV 1999, 367.

3 BT-Drucks. $14 / 56$.

4 BT-Drucks. 1 $\$ 1,69$. 
eincr fremden Sache, freilich nur insoweit, als zu deren Bcseirigung cin größerer Aufwand crforderlich ist.

Die Begründungen der Entwürfe weisen gleiche Argumentarionsmuster auf. Ausgangspunke ist der Unmut über eine uneinheiliche und zurückhaltende Rechrsprechung, die das Besprühen einer fremden Sache mir Graffici nicht stets als Substanzverletzung und damit als Beschädigung ansieht.' Die Zurückhaltung der Rechrsprechung erscheint im Lichte der von den Entwurfsverfassern gezeichneten Kriminalitätslagebilder unverständilich: "Graffiri-Schmierereien" nchmen demnach überhand und verleczen das »ästheuische Empfinden «." Graffici-Sprayer sind porentielle Vandalen, deren skriminelle Karriere schon im Keim erstickః werden soll.' Die dritte Gervait soll daher in die sicherheitspolitische Konzeprion des Geserzgebers eingebunden werden. ${ }^{8}$ Die Morive des Encwurfs sind polirisch en vogue; strafjuriscisch überzeugend sind sie nichr.

Der Gesetzgeber is aber gehalten, seine Gesetzesvorhaben zu begründen, um dic Allgemeinverbindlichkeit des Gesetzes zu verdeuclichon. Diese Verdeurlichung kann auf verschiedenen Ebenen erfolgen.

- Erfahrung mic der bisherigen Geseczespraxis (B.),

- normative Vertretbarkeit im Rahmen eines erkenntnistheoretischen, zumindest aber verfassungsrechelichen Maßsrabs (C.),

- empirische Grundlagen des Geserzesvorbabens (geselischaftliche Problemsenukturen und Folgewirkungen, unter D.),

- strafrechisdogmatische Folgen (E.).

Aus den Entwürfen zur Relorm der Sachbeschädigung spricht ein strafrechtliches Konzept, das den Rarionalitäısstandards dieser vier Ebenen niclt nur nicht genügt. Es ignoriert auch Altcrnaciven (F).

\section{B. Entwicklungslinien des Sacbbeschädigungs-Tatbestandes}

\section{Die Sachbeschädigung als schwere Substanzverletzung in den Straf- rechismodellen des 19. Jabrbunderts}

Nach dem im 19. Jahrhundert geformten Tatbestand des „Beschädigens und Zerstörens fremden Eigentums* sollen nur schwere Substanzverletzungen aks Straftaten erłaßc werden. Die Schwere der Substanzverletzung leiret sich aus der Höhe des Schadens oder der Bedcutsamkeit der fremden Sache für die Allgemeinhcit ab. Dabei orientiert sich die deutsche Geserzgebungsgeschichre am römischen Recht, das als Sachbeschädigung etwa das "Abhauen fruchtrragender Bäume, die Verletzung der Maucrn und Thore, die Verlerzung von Gräbern, von Wasserleitungen und Brunnen * ansah. ${ }^{9}$ Art. 366 des Enrwurfs eines Straf-Gesetz-Buches für das Königreich Württemberg setzte cinen Schaden von mehr als zehn Gulden voraus, um die Sachbeschädigung als Straftar ahnden zu können. ${ }^{10}$ Beschädigungen fremden Eigentums, die uncerhalb dieser Grenze lagen, wurden als "Polizeidelikt«, also als Ordungswidrig-

s Vgl. BT-Drucks. 1 4/872, BT-Drucks. 14/546, BT-Drucks. 14/569, jeweils S. 4.

6 So vor allem der Bundesratsenrwor und der Entwur der CDU/CSU-Bundestagstrakcion, B7-Drucks. $14 / 872$ und. $546,5.4$.

7 Ebd.

$8 \mathrm{Vgl}$. allgemein zu dieser "Bedrohung der Dritten Gewait durch irrationale Sicherheispolitik P. A. Albrechi, DRiZ 199\$, 326 ff.; sowic ders.. Kriminologie, 1999, S. 264 ff.

9 Vgl. A.F. Berner, Lehrbucli des deutschen Serafreclirs, Neudruck der 18. Auflage, Leiprig 1898, S.629.

10 Enrwurf eines Suraf-Geserz-Buches fur das Konigreich Wurtuemberg mil Moriven, 1836, S. 135 . 
keit angesehen. Die Sachbeschädigung im Strafgesetzbuch für das Königreich Baicrn verlangte einen Schaden von mehr als fünfzig Gulden." Für alle anderen Beeinträchtigungen fremden Eigentums - Besudeln, Beschmutzen, Entzichung der Sachewaren zivilrechtliche Ausgleichsmechanismen vorgesehen. ${ }^{2}$ Die Strafrechtsent würfe des 19. Jahrliunders und letzelich das Reichsstrafgesetzgebuch beschränken dic Strafbarkeir der Sachbeschädigung auf die Tathandlungen des Beschädigens und Zerstörens. Diese Tathandlungen grenzen die stralrechtsrelevante Eigentumsverleczung von zivilrechclich einschlägigen Eigentumsstörungen ab. Die Abgrenzung orienticrc sich am Schweregrad der Sachverlezung.

II. Die Sachbeschridigung in der Rechisprechung des Reichsgerichts: Variationen der Substanzalterierung

Jene Grenzziehungen mußten sich in der gerichrlichen Praxis bewähren und wurden dort alsbald gelockert. Die Entwicklung des Tatbestandsmerkmals »Beschadigen « in der Rechtsprechung vor allem des Reichsgerichts belegt diese Lockerung. Schon in der vielzitierter und -diskurierten Entscheidung des Reichsgerichts vom 19. Okcober $1885^{\text {"s }}$ - der Angeklagte hatte Bretrer aus einem Stauwerk herausgenommen und wcggeworfen - sind diejenigen Auslegungskriterien enthalten, welche die dogmatische Debacte bis heurc geprägt haben. Beschädigen bedcutete danach »nur eine solche Einwirkung auf die Sachc ( ... ), durch welche die Substanz der Sache alterierr, die

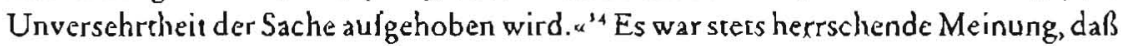
das Urteil des Reichsgerichts die Beschädigung als Substanzverletzung verstand. "So eindeutig ist der Wortlaut der Entscheidung indes richt. Behm weist zu Recht darauf hin, daß die Subsranzalterierung - also die bloße Veränderung im Zuscand der Sache den Kern des Beschädigungsbegriffs bildct. ${ }^{16}$ Die Aufhebung der Unversehrtheir - die Substanzverletzung - ist nur eine mögliche Variante der Substanzalterienng, für dic sich das Gericht im konkreten Fall entschieden hat. Der Ausschluß der *Brauchbarkeitsschmälerung * aus dcm Beschädigungsbegriff war ein einzelfallorienciertes Auslegungsmodell, das jederzeit zurückgenommen werden konnte. Im Begriff der Substanzalterierung sind mögliche Schwcrpunktverlagerungen im Tarbestand der Sachbeschädigung bereirs angelegt.

Dies zeigr sich in der Entscheidung des Reichsgerichts - einc Küchenhilfe hatte Kochtöpfe ihres Dienstherrn in den Rhein geworfen - vom 29. Januar 1904. ${ }^{17}$ Unter 8 eschädigung war auch die Brauchbarkeitsbeeinträchtigung zu verstehen. Substanzalccrierung konnte auch in der nachteilig veränderten Funktion der Sache bestehen. Der Funktionsaspekt erfährt sclaließlich in dem Urteil des Reichsgerichts vom 8./ 29.1.191018 weitere Differenzierung. Die Differenzienung eifolgt am Einzelfall. Fraglich war, ob das Bemalen eines Denkmals aus Marmor mit Farbe dem Tatbestand der Sachbeschädigung unterfällt. Die Auslegung der Tathandlung „Beschädigen a hat drei Schritte. Beschädigen meint - erstens - Substanzalterierung. Diese kann -

Is Stralgeserzbuch fur das Königrcich Baicm, i $813, \Lambda_{n}, 8_{3}$.

12 Vgl. W. Kasgl, JZ 199\%. 28 , (S, 2S9) mut wcitercn Nachwcisen.

is RCSe is, 27

14 RGS( 1$\}, 27\{28\}$

is Vgl. M. Maiwald, Der Zueignungsbegriff im System der Eigentumsdelikte, 1970, S. 122 If.; ders., Litc raturbericht (ZSiW 102, 319f.), dazu auch W. Kargl, JZ 1997, 283.

$16 \mathrm{Vgl}$. U. Bchm, Sachbeschädigung und Verunstalrung, 1984, S. II

17 RG GA $51,182 f$.

18 RGSt 43,204 
zwcitens - auch in einer Brauchbarkcitsminderung liegen. Wic der Uncersatz der Brauchbarkeitsminderung auszulegen ist, beurteilt das Reichsgericht nach dem allgemeinen Sprachgebrauch. Minderung der Brauchbarkeit kann danach - drittens auch die belangrciche Veränderung der Sache sein. Belangreich isı die Veränderung, wenn der Zweck der Sachc becinträchtig: wird."

Man kann an diesem „Dreisacz sehen, daß der Begriff der Substanzalierierung die Möglichkeir beinhalter, den Tatbestand auszudehnen, und daß von dieser Mögliclikeit auch Gebrauch gemacht wird. Ob eine Veränderung der Sache vorliegs, wird leczulich anhand des Zwecks bestimmt, welcher der Sache zugeschrieben wird. Nicht mehr nur das Ëigcntumsobjekt als solches ist das Schutzgut der Sachbeschädigung, sondern auch die soziale Funktion, die man dem jeweiligen Eigentumsgegenstand zumißr. Indem das Reichsgericht den mallgemeinen Sprachgebrauch " bemüh, um die Anwendbarkeic der Sachbeschädigung auf die Besudelung eines Denkmals zu prüfen, wird auf den Inhalr dieser sozialen Funktion verwiesen. Dieser Inhalt ist unbestimmt; er isc gesellschaftlich zeirbedingten, also historisch wandelbaren Bedürfnissen und Wertungen anhcim gestelle. Dic im Einzelfall gewählte Auslegung ist mit diesen sozialen Konstellationen eng verknüpft. Zutreffend weisc schon Tboss darauf hin, daß das Reichsgericht im Fall der topfwerfenden Küchenhilfe die "Strafdrohung wegen Sachbeschädigung benutzt, um die Arbeitsmoral zu fcstigen*.. ${ }^{20}$ In Fall der Denkmalsbesudelung hat das Reichsgericht dic Beeinträchtigung des Zwecks an ästhetischen Kriterien gemessen. Es hat besondere Eigenschafcen (Glanz, Rcinheit der Farbe, Bestimmtheit der Linien) herausgestellr, die das Denkmal zum ästhetisch wirkungsvollen Kunstwerk machen solken. Die Besudelung beeincrächrigce aus dieser Perspekrive die Zweckhaftigkeit des "Schönen«. Unverkennbar bleibr, daß die Ästherisierung des Eigencumsgegenstands nicht frei von Vorstellungen öffentlicher Orónung ist, die das Geriche normverdeurlichend vermitreln wollte. Albert Friedrach Berner formulierte den in der Strafrechtswissenschaft um 1900 herrschenden Zeitgeist: "Die nationale Unart bei den Deutschen, öffentliche Anlagen und Denkmäler zu bescliädigen, hangt zum Theil mit unentwickeltem Kunstsinn zusammen "." Nur teilweisc waren soiche "Beschädigungcn " vertretbar, wenn sic sich an als "undeursch $\propto$ verstandenen - "Nacktheiten der bildenden Kunse " vollzogen." Die "belangreiche Veränderung der Sache" in den Beschädigungsbegriff zu integrieren, ist hier niche nur professionelle Dognatik, sondern vor allem der Durchseczung herrschender Wertvorscellungen geschuldet, so ungenau faßbar diese - wie im Bercich der Kunst - auch sein mögen. Strafrechtsdogmatik ist Mitrel gesellschaftlicher Incegration, wobei bestehende Machrverhältrisse gegen Abweichung behauptet werden. Nochmal: In der Interpretationsentwicklung des Tatbestandsmerkmals * Beschadigen " gibr es keine Zäsuren von der "Subsınzverleczung über die Funktionsminderung zur Zustandsveränderung «, sondern nur begriffliche Schwerpunktverlagerungen innerhalb des Begriffs der "Substanzalterienung a. ${ }^{2}$ Dicse Schwerpunktverlagerungen umfassen bereits dicjenigen Kriterien, welche den dogmatischen und gesetzgeberischen Streic bis heute bestimmen. Der Tatbestand der Sachbeschädigung wird für den Wandel gesellschaftspolivischer Werte und Zielbesrimmungen geöffnet. Vorstellungen zur Ästhetik des Eigentumsobjekts sind Bestandteil dieses Wandels. In der Entscheidung des Reichsgerichts vom i 8. Dezember $1939^{24}$ schließlich bescimme

$19 \mathrm{RGSt}+3,204(205)$

20 Sielre P. Thoss, NJW $: 978,1612(1613)$.

21 A. F. Bemer (Fr. 9), 5.632

22 A. F. Berner, ebd, unter Verweis auf Jahn, Deutsches Volkstum, is $517, \mathrm{~S}_{18} \mathrm{ff}$

23 So auch im Ergebnis U. Bchm (Fn. 16). S. 40.

if RGSi 74,13 
sich die Substanzalierierung danach, ob von der Veränderung der Sache eine Gefährdung für die öffentliche Sicherheit und Ordnung ausgeht. Die Sachbeschädigung wird Bestandreil eines Gefährdungsstrafrechts, das für den autoritären Staat funktional war.

III. Beschädigen durch unbefugtes Plakatieren? Lebre von der Zustandsveränderung versus $B G H-$ Rechtsprechung

1. Die Lehre von der Zustandsveränderung als Beispiel verfassungsrechtlicher Kriminalisierungsgebote

Dogmatische Konsequenzen aufgrund negaliver Erfahrungen mit der politischen Disponibilität des Beschädigungsbegriffs blieben aus. Mitte bis Ende der siebziger Jahre entstehen mit den Fällen unbefugten Plakatierens dogmatische Problemkonstellationen, auf dic in der Rechrsprechung der OLG-Senare bereits entwickelte Kriterien angewandt weróen. Eigentumsobjekte sind danach bei der Veränderung ihrer äußeren Erscheinung beschädigt, wenn ihre Ansehnlichkeit durch die Veränderung beeinträchtigr wird. Das Kriterium der Anschnlichkeit rekurriert auf die Tradicionen äschecischer Werturteile im Tatbestand der Sachbeschädigung und bedingte in der Rechtsprechung schwer nachvollziehbare Wertungsungleichheiten. Ein plakasierter Brückenpfeiler"s soll cbenso unanselnnlich sein wie die plakacierte Sichtbetonwand einer Fußgängerunterführung. ${ }^{26}$ Das schlichte Grau des - strafrechrsdogmatisch berühmen - Telefonverteilerkastens ist in seiner Ansehnlichkeit durch das Anbringen von Plakaten hingegen nicht tangier. ${ }^{27}$ In der Literatur wurde erkannt, daß diese Wertungsunsicherheiten mit dem Rekurs auf das Eigentumsobjekr als Schut\%gut der Sachbeschädigung verbunden waren. Objekrive Bestimmungen der Substanzalterierungen waren unmöglich gemacht, wenn man auf dic Ästhetik der Sache abstellte; sie mußten insbesondere dann beinahe absurd wirken, wenn das Wertungsobjekt ein Gebrauchsgegenstand war. Lückenlose Strafbarkeit des unbefugten Plakatierens sollte sich aber aufgrund des verleczien Gestaltungsrechis des Eigentümers bezüglich des Eigentumsobjekts ergeben. ${ }^{28}$

Darin liegt die Ende der siebziger Jahre in der Strafrechrswissenschaft vollzogene Schwerpunkrverlagerung von dem Eigencumsobjekt zu dem Willen des Eigentümers, über die Sache nach seinem Belieben zu verfügen. Diese Schwerpunktverlagerung - in der Lehre von der Zustandsveränderung manifestiert - war nicht von Nachceil, weil sich der Begründungskontext der Tarbestandsinterprecation von einer tatbestandsimmanencen zu einer verfassungsrechtlich verankerten $Z$ weckbestimmung der Sachbeschädigung verlagert hatte und damit zumindest überprüfbare Kriterien in den dogmatischen Diskurs cingeführt wurden. Für die Auslegung des Straftarbestandes der Sachbeschädigung war damit jedoch niches an Verläßlichkeit und Vorhersehbarkeit gewonnen. Verändert hatte sich die Begründung der Strafbarkeitsausdehnung, richt die Tendenz der Ausdehnung selbst. In der Lehre von der Zustandsveränderung, die sich auf Eigentumsrechte aus Arı. ${ }_{4}$ GG und $\$ 90_{3}$ BGB berief, fanden die Rechte

2) OLG Hamburg NJW 1975,1981 I

26 OLG Karisrutie JZ 1975,642 ff.

27 OLC Karlsruhe JZ1978, 72

28 Vgi. F.C. Schroeder, Anmerkung zu OLG Karlsruhe. JZ 1978.72 f. 
des Verleızen Berücksichtigung. Die Grenzen des Strafrechts gegenüber dem Täter blicben jedoch undeurlich." ${ }^{29}$

Im Rekurs auf die Verfassung - auch dies eine Tendenz der siebziger Jahre - lag das Potential zu vermehrter Kriminalisierung: Das Verständnis der Verfassung als cincr objekriven Werteordnung, die dem Geserzgeber auch Strafpflichten auferlegen sollte, ist mit der Lehre von der Zustandsveränderung verbunden. Außer Betracht blieb und bleibr dabei, daß die strafrechtliche Reaktion auf die Rechrsverleczung als einem Eingriff des Staates in Freiheitsrechte selbst einem Maßstab unterliegen muß, der den Einsarz gerade des Strafrechts legitimicrt: Dieser Maßstab ist die Strafgeserzlichkeit. Gesetzlichkeit umfaßr cin materiell verstandenes Programm der Frciheirssicherung vor dem staatichen Zugriff. Das strafrecheliche Unrecht muB in vorhersehbarer Weise beschrieben sein, dic inkriminierce Handlung muß sich deuclich abheben von allräglichen Handlungen und von bestenfalls geringfügigen Normversiößen. ${ }^{30}$ Sachbeschädigung als strafrechlich relevantc Eigentumsverleczung muß sich von anderen Eingriffen in den Schutzbereich des Art. 14 GG und sonsiigen Störungen des Eigentums unterscheiden können. Gesetzlichkeir beinhalret insofern ein Srufenverhältnis in der Formalisienung des grundrechtlichen Eigentumsschutzes. Das Strafrecht greift nur dort ein, wo das mit der Rechtsverletzung verbundene Unrechu durch das Zivil-oder Ordnungswidrigkeitenrecht nicht mehr angemessen erfaßbar ist. In der Lehre von der Zustandsveränderung wird die Unterscheidung von strafrechtlichem, ordnungsrechtlichem und zivilrechtlichem Eigentumsschutz eingeebnet. Der strafrechtliche Eigentumsschutz wird umfassend. Mit der Strafgeseczlichkeit steht das nicht in Einklang.

\section{Eigentumsverletzung und Strafgesetzlichkeit in der Rechtsprechung des $B G H$}

Dem Beschluß des g. Strafsenats vom r 3 . November $1979^{\text {; }}$ komme das Verdienst zu, die ungeklärte Koexistenz der verschiedenen Kriterien zur Auslegung des Beschädigungsmerkmals - zumindest teilweise - aufgehoben zu haben. Dic belangreiche Veränderung der außeren Erscheinungsform wird aus dem Kriterienkanon ausgeschieden. Der dritte Schrirt des Reichsgerichss in der Auslegung des Merkmals "Beschädigen « wird zurückgezogen. Aus dem verlerzten Gestalcungswillen des Eigentümers solien sich keine strafrechrlichen Folgen ergeben dürfen. Damit ist mehr verbunden als nur eine Absage an die Lehre von der Zustandsveränderung. Erstmals wurden die Enrwicklungslinien der Sachbeschädigung insofern gebrochen, als die Judikatur Distanz zu der sozialen Siluation einnahm, in deren Zusammenhang der Tatbestand des $\$ 303$ StGB stand. Nicht das gesellschaftspolitische Ordnungsproblem - vermehre aufuretcndes unbefugres Plakatiercn öffenclicher Flächen - zu löser, begriff der Bundesgerichrshof als seine Aufgabe, sondern die Auslegung des Merkmals Beschädigen * nach dem Wortsinn sowic die Wiederherstellung der Balance von Strafrecht, Ordnungswidrigkeitenrecht und Zivilreche: "Die Vorschrift des $\$ 303$ StGB schürzt nicht, wie $\$ 1004$ BGB, die Belange des Eigentümers in umfassender Weise; strafrechclichen Schutz gewährt sie nur dem Interesse des Eigentümers an der

ig Überblick über dic Diskussion in Rechesprechung und Liccralur bei W. Küpsr. Bcsonderer Teil, 3. ncubearbeitete Auflage. 1999, S, 2$\} 2$ f.

30 Am Prinzip der Strafgeseczlichkeit orientierte Kritik an der Lehre von der Zusundsverandenung findet sich u. a. bei W. Kargl, JZ 1997, 2 S3 (S. 287 ff.) sowie bei M. J. Schmid, NJW/1979, 1590 (S. 1 583)

31 BCHSI 29.129 fi 
körperlichen Unversehrcheit seiner Sache a. ${ }^{22}$ Aus diesem Satz des Beschlusses spricht der Respekt des s. Strafsenats vor den materiellen Erfordernissen der Strafgesetzlichkcit. Gewonnen ist ein Auslegungsmodell, das vorhersehbare Kriterien erheblicher Eigentumsverlerzungen cnchält, die sich als uneingeschränkt justiciabel enveisen.

Die eingenommenc Distanz zu gesellschaftspolitischer Funktionalisierung der Sachbeschädigung hat sich in der Rechrsprechungspraxis nur mühsam durchgeseızt. Abweichende OLG-Entscheidungen - gerade in jüngster Zeit - belegen mitunter die fehlende Akzcpcanx des BGH-Judizes."

\section{Die "Ästhetik des Lebensgefühls" - Abschied von Traditionen des Strafrechts}

\section{Begründungserfordernisse}

Das Merkmal des Verunstaltens wird sowohl in dem Entwurf des Bunderaces bzw. der CDU/CSU-Bundestagsfraktion als auch in dem F.D.P.-Modell aus dem Zusammenhang der Substanzalterierung gelöst und verselbständigt. Die sozial situative Abhängigkeit der Sachbeschädigung - bisher durch Rechtsanwendung, nicht durch Gesetzgebung praktiziert - ist damic kodifiziert. Nicht die Bezugnahme auf Kriterien der Ästhetik ist dabei neu - die Entwicklungslinien der Sachbeschädigung sollten dies deurlich machen -, sondern die normativ gemeinten Legitimationsmuster, die in den Gesetzesentwürfen erkcnnbar werden und die das Verstänánis des $\$ 303$ StGB notwendig mirbestimmen.

\section{Rationalität des Begründungsdiskurses}

\section{a) Verfassungsrechticher Rabmen I: Eigentumsverletzung und Strafrecht}

Nochmal ein Blick zurück: Bisher war man es gervohnt, im Spannungsverhältnis von Eigentumsverletzung und gesexzlich besrimmem Strafrecht zu argumentieren. Man konnte darüber strcitcn, ob die mit dem Bemalen, Besudein, Besprühen oder Plakatieren fremder Sachen verbundene Verletzung frcmden Eigentums eine strafrechrliche Reakrion verlangte. Dieser Streit war möglich, weil sich überprüfbare und falsifizierbare Argumente gegenüber standen: aus Art. I 4 GG abgelcitetes Interesse des Eigentümers an umfassendem Schutz seiner Belange einerseits und strafgesetzlich enaßbares Unrecht der Sachbeschädigung andererseits.

\section{b) Verfassungsrechtlicher Rabmen II: Kunstfreibeit und Strafrecht}

Diese Argumente wurden um verfassungsrechrliche Problemstellungen ergänzc, als mit dem Fall des "Sprayers von Zürich " das Verhältnis von Strafgesetz und Kunstfreiheit berührt wurde. Das Bundesverfassungsgericht hatte argumentiert, der Schutzbereich der Kunstfreiheit erstrecke sich won vorneherein nicht auf die cigen-

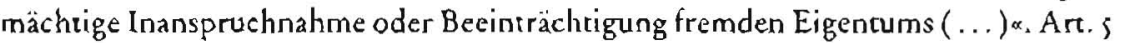

3. BGHSt 29, 129 (S. 133).

3) OLG Dusseldorf, NJW 1999, r 19g, dazu die kritische Anmerkung von U.Behm, NSiZ 1999, str. 
Abs. 3 Satz r GG gestatte einem "Künstler nicht schlechthin, sich über die Eigenrumsrechte anderer hinwegzusetzen. ${ }^{3+}$ Damit hatte das Bundesverfassungsgericht für die Rechtsanwendung der Sachbeschädigung lediglich die Berufung des Normadressaten auf den Schutzbereich der Kunst'reiheit ausgeschlossen. Von Befugnissen des Gesetzgebers, strafrecheliche Eingriffe in die Kunstfreiheit vorzunehmen, war im Zusammenlang mir Graffici nicht die Rede. Man wird hicr unterstellen müssen, daß Graffici unter den weiren Kunstbegriff des Art. s Abs. 3 Sarz I GG zu subsumieren sind. Es sind daher Schranken des Eingriffs zu diskutieren. Der Strafgesetzgeber wird sich dabei an dem verfassungsrechrlichen Erfordernis ausricheen müssen, daß Kriminalisierungsgebote mit Belangen öffentlicher Sicherbeit begründet werden müssen. Modelle öffentlicber Ordnung im Sinne jenes unbestimmten Rechtsbegriffs neiner Gesamtheit ungeschricbener Regeln für das Verhalten des einzelnen in der Öffentlichkeit, deren Beachtung nach den jeweils herrschenden Anschauungen als unerläßliche Vorausserzung eines geordncten staatsbürgerlichen Gemeinschafrslebens betrachter wird", genügen nicht, um einen schwerwiegenden, d. h. strafrechrlichen Eingriff in Grundrechre zu legitimieren." In diesem verfassungsrechtlichen Erfordernis findet sich dic aus den historischen Entwicklungslinien des Sachbeschädigungs-Tatbestandes ableitbare Erkenntnis wieder: Das Strafrecht, soll es in seiner Anwendung bercchenbar und voraussehbar sein, findet seine Grenzen in der Resiscenz gegenüber situativen, historisch wandelbaren Bedürfnissen gesellschafespolitischer Inregrarion. Das "Wahre, Schöne und Gute mag als wunerläßliche Voraussetzung gesellschaftlichen Zusammenhalts angeselien werden; als Legicimacion von Kriminalisierung taugt es jedenfalls nicht. Man darf von dem Strafgesetzgeber zumindest erwarten, daß die Strafwürdigkeic des zu kriminalisierenden Verhaltens so hinrcichend konturiert wird, daß der Anspruch des Sirafgesetzes auf Allgemeinverbindlichkeit erkennbar bleibr. Aber selbst diese - bcscheidene - Enwartungshalunng wird von den Geseczesentıüürfen entcäuscht.

\section{Begründungsverzerrungen der Strafgesetzgebung}

\section{1. "Life-style-Strafrecht"?}

Die vorgelegten Modelle zur Reform der Sachbeschädigung nchmen weder erkenntnistheoretische noch verfassungsrechdich verankerte Rationalitärsstandards der Gesetzesbegründung ernst. ${ }^{6}$ Deudich wird dies insbesondere an dem Entwurf des Bundesrates bzw. der CDU/CSU-Bundestagsfrakrion: Das Graffiti-Sprayen gilt gleichzeitig sowohl als Produkt verfallener Werte als auch als Moror voranschreitenden Werteverfalls. Graffiti ist das Symbol verlorengcgangener Moral, Ausdruck intolerablen gesellschaftsbclästigenden Lebensscils." Die verletzte „Äschetik des Lebensgefühls * bleibr als Bestrafungsgrund diffus. Man wird dies nicht nur für

34 BVeriG (Vorprolungsausschuß), Beschluß vom 19.3. 1984, NTW 1984. 1293 (1294. Sp. 1)

3s R. Scholy spricht in diesem Zusammenlang von der "Polizcilesligkeit" der Kunstfreiheit: "Soweit das Verhälenis von öffentlicher Ordnung und Kunst betroffen ish ist dic Kunstfrcihteit von vornherein und absoluz polizeifesta. Siehe Maury/Dürig/Scholz, Gundgeseiz-Kommentar, 7. Aufiagc, Stand Fcbruar 1999, Art.s Abs. 3 Rz. ss.

36 Dabei cxisticren beachatiche Erkenninisse, die dem Geserzgeber Rationalitatsseandards seiner Gesezzgebung vermitteln konnten. Vgl. nur G. Stazclielin, Strafgcserzgebung im Verfassungsstaat, 1998; I. Appel, Verfassung und Strafe, r 99g. Kricisch zur Einzelfallonentierung des Strágeserzgcbers nun auch K. Lassak, Begründungsdefizize in dèr aktucller Strafgesctzgebung, in: Institut für Kriminalwissenschaften und Rechtsphilosophic Frankfurt a.M. (Hrsb.), Irrwege der Strafgeserzgebung, 1999, S. 7s ff.

3) Daß die Morive der Encaurfe gesellschafeliches Gehor finden, ist dokumenticrt und analysiere bei wf 
eine verunglückte Formulierung halten dürfen, dic strukıurell an den Topos des "gesunden Volksempfindens erinnert. Hinter der Begründung steckt mchr und Grundsätzliches: Mit ilar kündigt sich der Abschicd all dessen an, was bisher unter Strafrecht verstanden wurde. Sie verortet das Strafrecht neu inmitten einer Gesellschaft, die durch prinzipielle Desorienticrung charakterisiert ist und deren Zusammenhalt sich durch die schleichende Auflösung des Nacionalscaass, wie sic mic okonomischer Globalisierung einerseirs und sicherheisspolitischer Privatisierung andererseirs sichtbar wird, verliert. Worin liegr das Neue an diesem neuen Strafrecht?

\section{Ordnung und Ambivalenz}

Die Geschichte des modernen Strafrechrs ist durch den dialekuschen Widerstrcit von Ordnung und Ambivalenz geprägt. Die um Problemiösung bemühte Moderne nutze Gesetzgebung und Rechtsanwendung als Ordnungsinstrumentarien, abweichendes Verhalten, das "Fremde" oder - in der Sprache der kricischen Theorie - das "Nichtidentischea zu erfassen und in die staatliche und gesellschafiliche Ordnung zu integrieren. ${ }^{18}$ Dabei entscehe mit der gefundenen Neurordnung auch neue Ambivalenz, die nach neuen Problemlösungsstrategien verlang̨. An den Encwicklungslinien der Sachbeschädigung kann man erkennen, daß die Auslegung des Tatbestandsmerkmals "Beschädigen schrittweise ausgedchnt wurde, um Antworten aul gesellschafiliche Problemlagen und mir diesen verknüpfte Strafbedürfnisse geben zu können. Für gegenwärtige Gesellschaften hat sich dic Funktion des Staates - und mit ihr die Funkcion des Strafrechts - innerhalb der "Dialekrik der Ordnung « verăndert.

\section{3. "Die Privatisierung der Ambivalenz"}

\section{a) Ambivalenz als allgemeiner Zustand}

Geselischaftlich wird die Veränderung der Lebenswels als beschleunigter Prozeß wahrgenommen, der jogliche Gewißheit in Zweifel zieht: wWas in einem Sektor des sozialen Lebens des Individuums Wahrheit ist, kann in einem anderen Sekror Irrrum sein. Was in dem einen Stadium der sozialen Laufbahn des Individuums als richrig angesehen wurde, wird im nächsten Stadium falsch ( . . ) $\alpha_{.}{ }^{99}$ Ambivalenz wird als allgemeiner Zuscand verscanden, der durch tradiertes staatliches Ordnungsinstrumentarium nicht mehr zu bewältigen schcinc. Dies führe nichr zur Toleranz des Ambivalenten, sondern zu neuem Unbehagen des Individuums, das nach Wegen sucht, jenes Unbchagen, jene potentielle Störung der Lebenswelt zu beseirigen. Damit bleibr diesc Suche der Idee verbunden, daß "persönliches Unbehagen ein im wesentlichen heilbarer Zustand ist “, daß die Ursachen des Unbelagens beeinflußbar sind und neurralisiert werden können. ${ }^{\circ}$ Es bleibe der private Glaube an die Herssellbarkeir von Ordnung. Im allgemeiner Zustand der Ambivalenz liegt es indes am Individuum,

Hasscmer, Neue Ansälze der Kriminaipolitik - Prävention durch Integration oder Repression, in: Ross. ner/Jehle, Kriminalität, Pravention und Kontrolle, 1999, S. 3 If.

3\& $\mathrm{Vgl}$ allgemeir zu dieser der Moderne immanenten xinstrumentellen Vemunft- Z. Bauman, Moderne und Ambivalenz, 1995, insbesondere S. $46 \mathrm{ff}$.

39 Berger v.a., Das Unbehagen in der Modernität, 1975. S. 162, zit. nach Z. Bauman (Fn. 38), S. 125. to Z. Buuman (Fn. 38), S. 254. 
die Wege zu dieser Ordnung selbst auszumachen und zu beschreiten. Damit gerät es in den Widerspruch, private Lösungen für Probleme finden zu müssen, an deren Komplexitäı die Gesellschaft insgesamt scheiterte. Die Diskrepanz von individuellem Wissen und gesellschaftlich norwendigen Einsichten ist zu groß, um die private Suche nach Oränung erfolgreich abschließen zu können." Das Individuum bleibo auf die Bercitstellung von Fachwissen angewiesen und versceht es als seine Pflicht, dieses Fachwissen abzurufen.

\section{b) Die Nachfrage nach Eindeutigkeit und das Normangebot der Beliebigkeit}

Zu verzeichnen ist daher eine Nachfrage nach irgendeiner Form der Eindeutigkeit, die zu befricdigen tradicrie gescllschafeliche Ordnungsstifter - selbse durch Prinzipienverlust gekennzeichnet - als Orte der Konzentracion sozialtechnologischen $W_{15}$ sens berufen sind: Wirtschaft, Medizin, Esorerik, Staat und Verwaltung, Kriminaljustizsystem. Dic Befriedigung der Nachfrage vollzieht sich vor dem Hintergrund der in ihrem Wichrigkeitsgrad eingeebneten sozialen Normen: Willensfreiheit und das Bedürfnis, sich bei McDonalds ungehindert vollscopfen zu können, körperliche Integrität und das Bestreben der »Wellnes-Bewegung ", den Körper physisch für die Herausfordenungen der Arbeirswelı zu rüsten, Eigentum und das Inceresse an ungestörtem Konsum inmitten parzellierter Welten von shopping-malls treten als gleichberechtigt schützenswerte Normen nebeneinander. ${ }^{11}$ In der prinzipiellen Orienrierungslosigkeic ist selbst das sogenannte Grundrecht auf Sicherheir zum Grundrecht auf ungetrübres Sicherheitsgefühl verkommen. Für das Kriminaljuscizsystem ist es bei der Sinnstiftung von Eindeutigkeit dann gleichgülig, ob der Raub oder die unsachgemäße Lagerung von Hackfleischbällchen kriminalisicre wird, wenn mit der Kriminalisierung die Nachfrage nach ciner - nur zufälligen - Eindeurigkeic gestillc wird. Das Vorhaben, die w Ästhecik des Lebensgefühls* zu einer strafrechrlich crfaßten Kategorie zu formen, ist Ausdruck dieser Gleichgülcigkeit und Resulcat nivellierter Sozialnormen.

\section{c) Nachpräventiv-postmodernes Strafrecht}

Strafrechuscheoretisch gewender bedeutet das: Das Strafrecht verliert an Nivcau, seine Karegorien diffundieren. Zu seiner Legitimation bedarf es weder Tärer noch Opfer, weder Rechtsgur noch Verfassung, weder präventive noch rcpressive Funktion, weder Zweckgedanke noch Vergeltungstheorie. ${ }^{41}$ Was entsceht, ist ein - so die Formulierung Wolfgang Nauckes - nnach-präventives $\alpha$, im schlechten Sinne postmodern konturloses Regelgebilde. ${ }^{44}$ Das Strafreche steht möglicherweise vor dem letzen historischen Schritt seincs Wandels: Vom Rech, das den Staat in seinem Zugriff auf Freiheirsrechte begrenzt, über die Regeln, welche zur Bekämplung von Verbrechen und Vergehen funkcional sind, zu einer "Störungsbeseitigungsordnung*, in der alles und gar nichts kriminalisiert werden kann, je nachdem, welche Anrworten das in der privarisierten Ambivalenz überforderte Individuum verlangt. In den Modellen zur

41 Vgl. au dem problemalischen Verhalenis von *Wissen, Verarauen und Reclix H. Rossen-Stadeleld, KrikV 1999. 223 If.

42 Das Angebor der Normenbelicbigkeit wird cindruckswoll beschricben bei Z. Bauman, Flancurc, Spicler und Tourister, 1997, S. 17: wAlso laße uns dicse W/cit, entlaster von cingebilderen Verpflichiungen und falschen Pflichen, feiern. Wenn universale Prinzipien und absolute Wahrheiten crse cinntal vergeudec oder aus der Made getommen sind, dann spielt es keine Rolle nehr, welche persönlichen Prınzipicr man unterstüere (...) und befolgt (...). .

4) Vgt. W. Hassemer (Fn. 37), S. 9 [l.

44 W. Nauckc, KritV 1999, 319 ff. 
Reform des Sachbeschädigungs-Tarbestandes soll diese «Störungsbeseitigungsordnung zum Ausdruck gelangen. Unrechtsbegründungen der Sachbeschädigung, gleichgültig, ob sie Eigentümerinteressen oder Strafgesetzlichkeit zum Kern haben, werden mic der Strafwürdigkeit von "life-style-Verfehlungen a jeglicher Ernsthaftigkeic beraubc.

\section{Empirische Zerrbilder der Jugendkriminalität}

Aber nicht nur normativ nimme der Geserzgeber eine nachgerade unüberbrückbare Distanz zu den Legitimarionsanforderungen der Strafgeselzgebung ein. Auch empirisch ist diese Distanz sichtbar. Die vorgelegten Entwürfe gehen von zwei nicht belegbaren Annahmen aus: "Graffiti-Schmierereien nehmen quantitaciv überhand " und: Graffiti stehen bei Jugendlichen am Beginn einer kriminellen Karriere $\alpha$, bilden eine "Einstiegsdroge für abweichendes Verhalten. Diese Annahmen überschätzen den Aussagewert von Justizstatistiken und verkennen die kriminologischen Spezifika abweichenden Verhaltens bei Jugendlichen.

Der Aussagewert von Justizstatistiken ist deutlich zu relativieren. Sie eignen sich in erster Linie als "Geschäfısstaristiken*, als Bilanz sozialer Kontrolle." Selektionsmechanismen im Trichtermodell des Kriminaljustizsystems erweisen sich als zu dominant, um verallgemeinerungsfähige Aussagen über die Entwicklung von Kriminalităt in einer Gesellschaft treffen zu können. Wenn überhaupt Aussagen zur Kriminalitärsentwicklung getroflen werden sollen, so ist dies nur aufgrund einer Relation zwischen absoluten Zahlen bei Tatverdächtigen, Angeklagten, Abgeurteilten und Strafgefangenen und der Gesamtbevölkenung möglich. Für die polizeiliche Kriminalstatistik ergibr sich bci der Sachbeschädigung cine Tarverdächrigenbelastungsziffer zwischen 0,15 (1990) und o, ig \% (1997). ${ }^{46}$ Es besteht kein AnlaB, die Verletzung fremden Eigentums zu dramacisieren. Von einem quantitativen $»$ Überhandnehmen « der »Graffiti-Schmierereien« läßr sich nur sprechen, wenn man das weitc Feld alleäglicher Vorurteile nicht verläßc.

Jugendspezifische Verfehlungen sind im wesentlichen durch Eigentumsdclikte bestimm und haben - gemessen am Schaden - eher Bagatellcharakıer. ${ }^{77}$ Vor diesem

4s Siche P-A. Albrechı, Krıminologic, 1999, S. 147.

${ }_{46}$ Vgl. Bundeskriminalame (Hrsg.) Polizeiliche Kriminalstacisrik Berichtspahre 1990-1997, Tabcilc ol.

1. Absolute Zahien
\begin{tabular}{|l|l|l|l|l|l|l|l|}
\hline 1990 & 1991 & 1992 & 1993 & 1994 & 1995 & 1996 & 1997 \\
\hline 91.178 & 110.182 & 121.128 & 129.944 & 136.669 & 148.394 & 155.439 & 162.155 \\
\hline
\end{tabular}

2 Tatverdächrigenbelasnugs'iffer (vgl. zu deren Bedeurung fur die Analyse von Juscizstatistiken, P.-A. Albrechi, Kriminologic, 1999, S. 145 ff.

\begin{tabular}{|l|l|l|l|l|l|l|l|}
\hline 1990 & 1991 & 1992 & 1993 & 1994 & 1995 & 1996 & 1997 \\
\hline $0.15 \%$ & $0,17 \%$ & $0,18 \%$ & $0,16 \%$ & $0,17 \%$ & $0,18 \%$ & $0,19 \%$ & $0,19 \%$ \\
\hline
\end{tabular}

3. Tatverdächugenbelasmungsziffer Uür Sachbeschädigungen auf Straßen, Wegen und Platzen

\begin{tabular}{|l|l|l|l|l|l|l|l|}
\hline 1990 & 1991 & 1992 & 1993 & 1994 & 1995 & 1996 & 1997 \\
\hline $0,012 \%$ & $0,012 \%$ & $0,013 \%$ & $0,011 \%$ & $0,012 \%$ & $0,014 \%$ & $0,015 \%$ & $0,015 \%$ \\
\hline
\end{tabular}

47 P.-A. Albreche, Jugendsirafreche, 2- Auilage, S. 9 
Hintergrund sind die vorgetragenen Rezepte einseitig, setzten auf Moralisierung und Erziehung, vertrauen auf die harte, zupackende Ausgrenzung des Störenden. Versucht wird eine Normverdeuclichung, die im allgemeinen Zustand der Ambivalenz mißlingen muß. Wo soziale Normen in ihrem Bedeutungsgehalt eingeebner wurden, erscheint die Zuschreibung von Nornverlexzungen zufällig und vergänglich. Für jugendliche Subkulouren gewinnt das eingeforderte Modell sozialkonformen Verhalrens damic wenig an normativer Überzeugungskraft. ${ }^{+\gamma}$ Das von der "Störungsbeseitigungsordnung statuierte Gesellschafesmodell ist nur eine von viclen Wahlmöglichkeiten, die eigene Lebenswelt so oder ganz anders zu gestalten. Graffici-Sprayen steht schon deswegen nicht am Beginn einer skriminellen Karrierek, weil die zu verzeichnende Episodenhafrigkeit abweichenden Verhakens Jugendlicher in Zeiten der Normenunklarheit noch verstärki wird." Polemisch formulicrt: Was hcure als Verletzung des Eigentums verstanden wird, ist morgen möglicherweise erlaubte Kunst. Was morgen erlaubt ist, wird für den eigenen Weg individueller Sinnstiftung auch uninteressant. Erst die Anwendung des Strafrechts auf mlife-style-Verfehlungen a droht die Zuschreibung der Störereigenschalt zu verfestigen und den Bruch zwischen Gesellschaft und Individuum zu bcsiegeln.

\section{E. Dogmatische Folgewirkungen}

Die Verzerrungen und Defizire der Entwurfsbegründung schlagen sich in einer Tatbestandsformulierung nieder, die kaum und nur unter großen Schwierigkeiten justitiabel sein dürfce.

Verunstalcen ist dic belangreiche Veränderung des äußeren Erscheinungsbildes der Sache. Als belangreich wird diejenige Veränderung verstanden, welche nur durch einen nichc zu vernachlässigenden Aufwand zu beseitigen isc. Den Entwurfsverfassern isı bewußt, daß das vorgeschlagene Merkmal Abgrenzungsschwierigkeiten zu "bagateil haften Veränderungen" aufwirft. so Bloßes Beschmutzen oder Bemalen des Gegenstandes soll - nach den Entwürfen des Bundesrats und der CDU/CSU-Bundestagsfraktion - nicht umlaßt sein, um die Strafbarkeit nicht zu wüberspannen*." Diese Abgrenzung düıfte mißlingen, da sie - wie die Entwicklungslinien des Tatbescandes deutlich machen - auf Kriterien ästhetischer Werturtcile angewiesen blcibr, wenn es um die Veränderung von Gegenständen geht, denen der Eigentumsberechtigte ausschließlich ästherische Zwecke zuschreibt. Ob der Gegenstand nur wbemalt « oder bereits "verunstalcec* wurde, hängt von der künstlerischen Einschätzung des Gestaltungsbercchtigten ab, die zum Gegenstand der tatrichterlichen Beweiswürdigung gemacht werden müßte. Zu Recht urteilte der Bundesgerichtshof, daß es an allgemeingültigen Maßstäben fehle, um den ästhetischen Wert eines Gegenstandes beurceilen zu können.' ${ }^{\prime 2}$ Ästhetik ist keine Kategorie, die intersubjektiv verbindlich vermitrelt werden könnce. Es ist nicht Aufgabe des Strafrichters - so der Bundesgerichtshof -, Maßstäbe von Ästhetik durch Urteil festzusetzen." So bliebe nur, die einseirige Zweckbestimmung des Gcgenstandes durch den Eigentümcr für allgemein-

48 Vol. P.-A. Albrecht, Kriminologie, S. 39.

$49 \mathrm{Vgl}$ allgemein zur Episoderhafigkeit von Jugendkriminalieat P.-A. Albrecht, Jugendstrafreche, 2. Aufl., S. 2016 .

go BT-Drucks. I $4 / 546$, S. 6 und $14 / 872$, S. s.

s। Ebò.

\$2 BGHST 29, 129 (134).

s) Ebd. 
verbindlich zu erklären. Diese Zweckbestimmung ist nach außen indes nicht er-

kennbar. Walter Kargl stellt dazu richtig fest, daß über die Tatbestandsverwirklichung dann der Zufall entscheide. ${ }^{44}$ Ein solcher Tatbestand sreht zu dom Prinzip der Strafgeseczlichkeit in Widerspruch.

\section{F. Zusammenfassung und Alternativen}

\section{Verfehlte Rationalitätstandards}

Insgesamt: Die vorliegenden Reformentwürfe rekurrieren auf Entwicklungslinien der Sachbeschädigung, die den Tarbestand gesellschaftspolitisch funktional ausdehnten. Aus den damit verknüpfien Negativerfahrungen dogmatischer Wertungsunsicherheit werden keine Konsequenzen gezogen. Im Gegenteil: Die strafjuristische Distanz, die der Bundesgerichtshof zu der gesellschaftlichen Situation der Sachbeschädigung hergestellt hacre, soll endgültig zurückgenommen werden. Mit dem erneuten Rekurs auf gesellschaftspolitische Funktionalitäı entsteht in dem gegenwärrigen Zustand allgemeiner Ambivalenz ein Strafrecht, das überprüfbare Rationalitäıscandards zu verlieren droht. Auf die Antinomien tradierter Begrünờungsansätze - Rechtsgut, Verfassung, Gesctzlichkeit - kommı es nicht mehr an. Es drohı die Erablierung einer $*$ Störungsbeseitigungsordnung $\alpha_{\text {, }}$ in der alles und gar niches kriminalisiert werden kann. Dícse finder in der "Ästhetik des Lebensgefühls" als strafrechtswürdiger Kategorie ihren Ausdruck. Die kriminalpolitische Dramatisierung des "Graffiti-Phänomense ist empirisch nicht substantiiert. Die polizeiliche Kriminalstatistik belegt seit 1990 Tatverd̈ächcigenbelastungsziffern auf niedrigem Niveau mit geringen Schwankungen. Die Episodenhaftigkeit von Jugenddelinquenz - verstärkt durch die Unbeständigkeit sozialcr Normen - wird verkannt. Im dogmatischen System ist der mit dcm unbestimmten Merkmal "Verunstalten * angerichtece Schaden groß.s"

Nach im Jahre 1982 harte der Rechrssausschuß des Deurschen Bundestages - bei nur einer Gegenstimme - empfohlen, sogar aur die Einführung eines Bußgeldratbestandes über das Verunstalien von baulichen Anlagen oder gemeinnürzigen Sachen zu verzichten ( $\mathrm{E} \$ 1$ 1 $8 \mathrm{a}$ OW $\mathrm{W} G$ ). Den Fraktionen der CDU/CSU und der F.D.P war selbst der Bußgeldtarbestand zu unbestimmt. ${ }^{16}$ Sie hätten es bei dieser Einsicht belassen sollen.

\section{Bessere Alternativen}

Um es zu verdeutlichen: Es ist klar, daß cs sich bei dem Besprayen fremder Sachen mit Graffiti um eine Störung des Eigentumsrechts handelt. Die Rechtsordnung hält jedoch in anderen Reclısgebiecen angemessene Reakrionsmöglichkeiten auf diese Störung bereit. Deren Effizienz sollte kritisch überprüft werden. Dies gilt insbesondere für den zivilrechtlichen Bescitigungs- und Unterlassensanspruch gemäß $\$ 1004$ Abs. I BGB. Von zivilprozessualen Beweisproblemen einmal abgesehen, er-

i4 Kargl, JZ 1997, 283 (S. 287)

is Im Ergebris ablehnend auch U. Belum, SeV 1999, 567 (S. 572)

s6 BT-Dructes. 10/5083, S. 23 . 
streckt sich der Inhalt des Beseitigungsanspruchs immer noch aul die Abstellung der Einwirkung für die Zukunft, nicht jedoch auf die Herstellung des früheren Zustandes durch Folgenbeseitigung. Die Unterscheidung von Beseirigung der Beeinträchrigung und Schadensersarz wird im zivilrechtichen Schriftum und in der Rechtsprechung niche cinheitlich vorgenommen." Das Zivilrecht müßte für Regelungen geöffnes werden, die eine Verurcilung zur Leistung, also zur persönlichen Beseitigung der Eigentumsstörung unıcr Nutzung eigener zcillicher und materieller Ressourcen ermöglichen.

Neben dic rechtliche Reaktion auf die Störung des Eigentums sollten kommunalpolitische Strukturverbesserungen treten. Graffiti ist vor allem ein großstädtisches Problem. Jugendliche wählen Ausdrucksformen, die ebenso wie dic sozialen Normen, gegen die sie sich richten, besrändigem Wandel unterworfen sind. Längst können in den Jugendszenen der Großstädce Tendenzen ausgemachı werden, die von dem "Abschwung der "Graffiti-Kulcur zeugen. Vor dem Hintergrund sich verändernder Jugendkulcuren kommr der Geseczgeber zu spät. Längsc hat zudem die Kommunalpolicik reagierc und öffentliche Flächen geschaffen, auf denen Jugendliche legal "sprühen * dürfen. Bizarre Farbgebilde in den U-Bahn-Starionen Frankfures und Bcrlins belegen beispielsweise diese legalen Aktivitäten. Graffici-Probleme - in urbanen Strukturen entstanden - verlieren sich auch rasch in großstädrischer Schnelllebigkeit und werden im kommunalpolitischen Umfeld an besten bewäligt. Die Entwurfsverfasser haben diese Wirklichkeit freiheirlicher Problemlösung völlig aus dem Blick verloren.

Heike Vaupe?

\section{Die Familienrechtsreform in den fünfziger Jahren im Zeichen widerstreitender Weltanschauungen}

Seit Gründung der Bundesrepuhlik Deutschland wurde das Familienrecht grundlegend geänderl. Die wohl einschneidendsten Eingriffe in die ursprüngliche Fassung der einschlägıgen Vorschriften erfolgten in den füniziger Jalıren dicses Jahrhundens. Sie waren durch die grundgeselzliche Verankerung des Prinzips der Gleichberechtigung veraniabi.

Die Jahre nach Inkrafitrelen dles Grundgeselzes waren so geprägt vom Kampf um eine angemessene Umsetzung des Gleichheilssabzs in das einfache Recht. Im Miltelpunkı der Familienrechtsrefor in standen dabeı die Vorschrifien des persönlichen Eherechts sowie das Kindschaftsrecht in der Fassung von 1900.

Die Arbeit analysiert am Bcispiel dieser Bestimmungen, welche Schwicrigkeiten der Durchführung des Gleichberechtigungsgrundsalzes im Wege slanden. Dabci arbcitcl die Verfasscrin nichi nur den wechselvollen Gang bci der Verwirklichung des Gleichbercchtigungsgrundsalzes im Famblsenrecht scil 1948 auf, sondern mache auch die viclfältigen Ursachen, Motive, demoskopischen Gegebenheiten und Weltanschauungen deurlich. die in das Gesel_gebungsverlahren Eingang gefunden und seinen Forlgang bestimnt lyaben.

1999, 236 S., brosch., 72, - DM. 526. - ôS. 65.50 sFr, ISBN 3-7890-6396-7

(Schriften zur Gleichstellung der Frau, Bd. 22)

\section{NOMOS Verlagsgesellschaft}

76520 Baden-Baden

37 PalanduBassenge, Bürgerliches Gescrabuch, 38. Auflage, $\$ 1004, R 722$. 